\title{
Kin recognition and perceived facial similarity
}

\section{Florian Hansen}

Lisa M. DeBruine

Iris J. Holzleitner

Anthony J. Lee

Kieran J. O'Shea

\section{Vanessa Fasolt}

King's College, Department of Psychology, London, UK

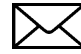

University of Glasgow, Institute of Neuroscience and Psychology, UK

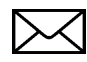

University of Glasgow, Institute of Neuroscience and Psychology, UK

University of Stirling, School of Psychology, UK

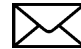

University of Glasgow, Institute of Neuroscience and

Psychology, UK

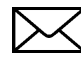

University of Glasgow, Institute of Neuroscience and

Psychology, UK
Facial similarity between individuals informs kinship judgments in third-party kin recognition. Indeed, one study found that similarity and kinship judgments encapsulate the same information (Maloney \& Dal Martello, 2006). Yet, another study found that this is not the case when comparing adult face pairs of different sex (DeBruine et al., 2009). We replicated these studies to further clarify the role of facial similarity in kin recognition. We recruited 318 raters, who were shown 50 sibling pairs and 50 age- and sex-matched unrelated pairs ranging from 3 to 17 years old. Each rater was randomly assigned to make either kinship judgments ("related" or "unrelated") or similarity judgments (scale from 0 [not very similar] to $\mathbf{1 0}$ [very similar]). The threshold model found that performance in both tasks was equally accurate, with participants detecting child siblings in the kinship task above chance and giving significantly higher similarity ratings to siblings in the similarity task. In both tasks, opposite-sex siblings were perceived to be siblings less often than same-sex siblings, and judgments of unrelated face pairs were not affected by the sex of faces. Conversely, the effect of age difference within pairs of faces differed for the two tasks: a greater age difference decreased all kinship judgments, but only decreased similarity judgments of siblings, not unrelated pairs. In line with DeBruine et al. (2009), these findings suggest that similarity and kinship judgments are highly correlated but not strictly synonymous. The OSF Pre-registration Challenge for this project can be found at osf.io/ps9hy and the data at osf.io/sef9k.

\section{Introduction}

Social interactions are influenced by a myriad of factors, one of them being facial similarity. Facial similarity to oneself can lead to an increase in prosocial behavior, such as an increase in trusting behavior in a trust game (DeBruine, 2002), or an increase in an individual's contribution to the group in a public goods game (Krupp, DeBruine, \& Barclay, 2008). Moreover, self-resemblance increases positive attitudes toward children (Bressan, Bertamini, Nalli, \& Zanutto, 2009; DeBruine, 2004b; Platek et al., 2004), trustworthiness perception of opposite sex faces (DeBruine, 2005), and attractiveness perception of same sex faces (DeBruine, 2004a). These behaviors and perceptions are consistent with the notion that facial cues are used when making kinship judgements (see DeBruine, Jones, Little, \& Perrett, 2008 for a review).

Perceived similarity is generally based on comparing a subset of features from a general pool of facial cues for similarities or differences (Tversky, 1977), allowing the observer to derive a similarity "aggregate." When making judgments about facial similarity and relatedness, the same general pool of cues is used. As outlined by Maloney and Dal Martello (2006), this process can lead to two possibilities. On the one hand, different subsets of features and different combinations of features might be used to derive a kinship aggregate and a similarity aggregate, meaning that similarity and kinship decisions are made independent from each 
other, or have only little influence on each other. For example, features differing owing to sex and age might inform similarity judgments, but may be irrelevant in making kinship judgments. On the other hand, it is possible that a similarity aggregate holds all the information used to make kinship decisions, meaning that a certain similarity aggregate forms the threshold for a positive or negative kinship judgment.

Two studies have looked at the extent to which similarity judgments and kinship judgments of pairs of faces overlap, or are synonymous, and how these judgments correspond with actual relatedness, with differing conclusions (DeBruine et al., 2009; Maloney \& Dal Martello, 2006).

Maloney and Dal Martello (2006) used 30 pairs of facial photographs of children (17 months to 15 years of age). One-half of these 30 stimuli pairs were related and one-half were unrelated, with age and sex distribution matched between the two sets. They then familiarized 64 participants with their stimuli, and assigned one-half of the participants to the kinship task and the other one-half to the similarity task. In the kinship task, participants judged whether a shown pair was related or not. In the similarity task, participants judged how similar a pair looked on a scale from 0 to 10 (the higher the score, the more similar the pair). In the kinship task, participants were made aware that one-half of the pairs shown are actually related, whereas participants in the similarity task did not get any information about relatedness status. Maloney and Dal Martello (2006) found that pairs receiving higher similarity ratings were also judged to be siblings more often, whereas pairs receiving lower similarity ratings were judged to be siblings less often. Moreover, actual relatedness was a strong predictor of similarity ratings as well as kinship judgments, albeit similarity ratings tracked actual relatedness more closely without reaching a significant difference. These results lead the authors to conclude that "perceived facial similarity of children is little more than a graded kin recognition signal."

DeBruine et al. (2009) replicated this study using face photographs of adult siblings (16-46 years of age). They used two image sets, one consisting of 16 female nonidentical (dizygotic) twin pairs and 16 age-, ethnicity- and sex-matched unrelated control pairs, and one set consisting of 10 sibling pairs and 10 age-, ethnicity-, and sex-matched unrelated control pairs. Two versions of each face stimulus were created, one showing hair and clothing, the other masking hair and clothing. One hundred eighteen raters viewed both the twin set and the sibling set, but raters only saw one version of the face stimuli (i.e., masked or not masked), and completed only one of the tasks (i.e., the kinship task or the similarity task). Similarity and kinship judgments were correlated, with the correlation being lower for non-twin sibling pairs than twin pairs. Similarity ratings were strong predictors of a pair being actually related, but significantly less so for the unmasked non-twin sibling set. This finding suggests that the similarity judgments of adult faces varying in age and sex may at least partly be based on cues other than relatedness. Based on this result, the authors explored the effect of sex on similarity ratings. They found that unrelated same sex pairs received higher similarity ratings than unrelated opposite sex pairs. In contrast with Maloney and Dal Martello (2006), DeBruine et al. (2009) did find a significant difference in task accuracy, but only in the unmasked sibling set. Here, kinship judgments were more accurate at discriminating actual siblings and unrelated pairs than similarity ratings. The authors suggested that explicit kinship judgments are more effective in detecting actual siblings than similarity ratings, at least in a set of adult siblings of different ages and sex compositions. However, age and sex differences within the pairs were confounded, because same sex and opposite sex pairs differed in age differences. Nevertheless, the authors interpreted their findings as evidence that similarity judgments convey some information that is not conveyed in kinship judgments when comparing adult face pairs.

The current study replicates Maloney and Dal Martello's (2006) design using a larger number of child sibling pairs (100 pairs in total, 50 siblings and 50 matched controls). Based on previous findings we hypothesized that:

1. Threshold similarity judgments do not provide identical information to kinship judgments.

2. Sex differences negatively influence similarity, but not kinship judgments.

3. Age differences negatively influence similarity, but not kinship judgments.

\section{Methods}

The methods for this study were pre-registered in the Open Science Framework (OSF) Pre-registration Challenge at osf.io/ps9hy. The planned analysis script, as well as details about the hypotheses, stimuli, procedure, and exclusion criteria are all available at this site. All procedures and analyses described here follow this pre-registration exactly. An additional model has been added into the analysis section. This model tests whether the effects found for each task are significantly different between tasks.

\section{Stimuli}

Stimuli were collected from children visiting a local science center who volunteered to take part in a study 
of facial cues of family relatedness. Parental consent and child assent were obtained from each child to use their face photograph in studies of family resemblance. Children were photographed with a neutral expression, with hair tied back and any glasses, scarves, or hats removed. The specific procedures for image collection are available on the OSF at osf.io/bvtnj.

From a set of approximately 2,000 images of individuals of varying ages, sex, and relatedness, we algorithmically chose the maximum number of sibling pairs fitting a number of criteria. Both siblings were required to be genetically full siblings (but not twins) and under the age of 18. An additional required condition was that an age-matched (within 1 year), ethnicity-matched, and sex-matched foil image was available to use from family units that were not represented elsewhere in the image set. Specifically, the two individuals in each sibling pair are related to each other, but not to any other individual in the set, and all individuals in unrelated pairs are related to no individuals in the set.

This procedure produced 50 non-twin sibling pairs and 50 matched unrelated pairs. In each group, 28 pairs were same sex pairs (13 boy pairs, 15 girl pairs), and 22 pairs were opposite sex pairs. The individuals ranged from ages 3 to 17 years old (mean age, $9.44 \pm 2.92$ ) and the age difference between individuals in a pair ranged from 0 to 7 years (mean age difference, $2.96 \pm 1.64$ years).

\section{Procedure}

Raters completed the experiment online at faceresearch.org on their own computer. Raters were recruited through social media and social bookmarking sites. Each rater was randomly assigned to either the kinship or similarity judgments task.

For both tasks, raters were told that they would view 100 pairs of faces. Raters in the kinship task were also told that some of the pairs were siblings, whereas relatedness was not mentioned in the instructions of the similarity task. For the kinship task, raters were then asked to judge whether any shown pair was related by clicking on buttons labeled "unrelated" or "related." For the similarity task, participants were asked to rate each pair for similarity on a scale from 0 (not very similar) to 10 (very similar). These procedures are identical to previous work (DeBruine et al., 2009; Maloney \& Dal Martello, 2006).

\section{Raters}

We specified in our pre-registration that we would collect 100 raters in the similarity task and 100 raters in the kinship task to have sufficient power to detect even a small effect. The study ran online and there was a high amount of traffic on the website; therefore, we overshot this target before taking the study offline again. We decided to include all 318 raters (143 raters in the kinship task and 175 raters in the similarity task) in the following analysis to increase power and use all available data. Crucially, running the analysis with 318 raters or the pre-registered 200 raters did not change the results. All data are available on the OSF at osf.io/sef9k.

Stimuli were rated for kinship by 81 men (mean age, $29.94 \pm 11.67$ years) and 61 women (mean age, $28.59 \pm 12.21$ years), whereas 91 men (mean age, $28.24 \pm 12.84$ years) and 83 women (mean age, $29.31 \pm 12.34$ years) rated the stimuli for similarity. Two raters did not indicate their gender.

\section{Results}

\section{Kinship task}

We used a logistic mixed model to predict relatedness judgments from actual relatedness (effect-coded as related $=+0.5$ and unrelated $=-0.5$ ), sex difference (effect coded as same-sex $=-0.5$ and opposite-sex $=+0.5$ ), age difference (centered), and all possible interactions in the kinship task. Analyses were conducted in $\mathrm{R}$ version 3.5.0 (R Core Team, 2017) using the packages lme4 version 1.1.17 (Bates, Mächler, Bolker, \& Walker, 2015) and lmerTest version 3.0.1 (Kuznetsova, Brockhoff, \& Christensen, 2016).

The analysis revealed a main effect of relatedness, $\beta=1.09, S E=0.16, z=6.8, p<0.001$, odds ratio $=2.97$, whereby related pairs received a higher proportion of "related" judgments than unrelated pairs. Furthermore, there was a main effect of age difference within a pair, $\beta=-0.15, S E=0.05, z=-2.95$, $p=0.003$, odds ratio $=0.86$, whereby the greater the age difference within the pair, the lower the proportion of "related" judgments they received, regardless of actual relatedness (Figure 1). The main effect of sex difference was not significant, $\beta=-0.31, S E=0.16, z$ $=-1.95, p=0.051$, odds ratio $=0.73$, but was in the direction of same sex pairs receiving a higher proportion of related judgments than opposite sex pairs. There was no interaction between relatedness and sex difference, $\beta=-0.56, S E=0.32, z=-1.75, p=0.08$, odds ratio $=0.57$, relatedness and age difference, $\beta=-0.13$, $S E=0.1, z=-1.28, p=0.199$, odds ratio $=0.88$, sex difference and age difference, $\beta=-0.1, S E=0.1$, $z=-0.96, p=0.335$, odds ratio $=0.90$, or relatedness, sex difference and age difference, $\beta=-0.29, S E=0.2$, $z=-1.46, p=0.143$, odds ratio $=0.75$. 


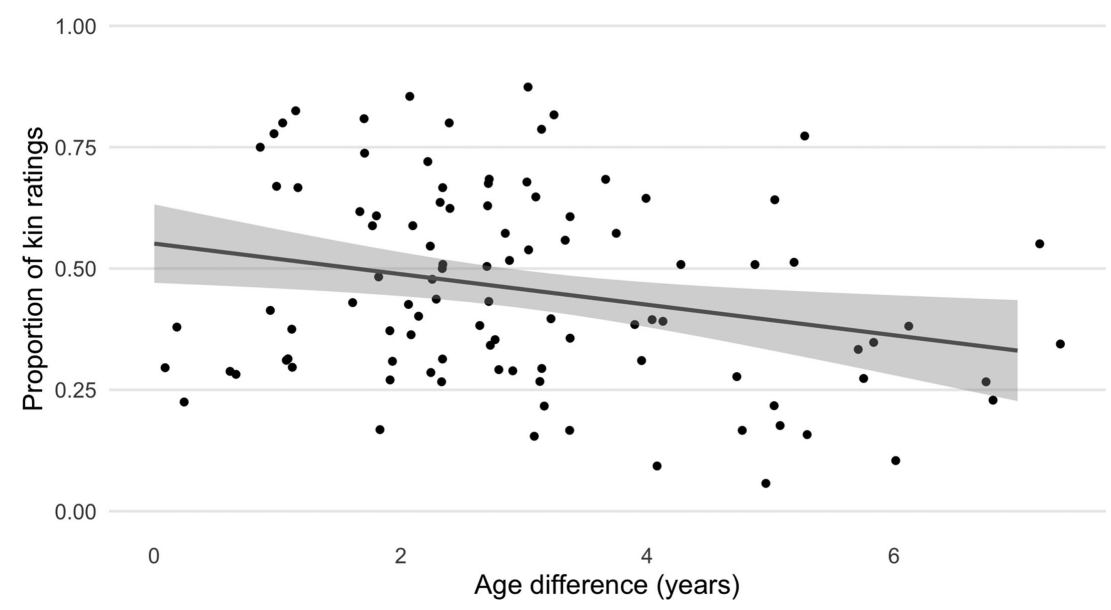

Figure 1. The main effect of age on proportion judged as related.

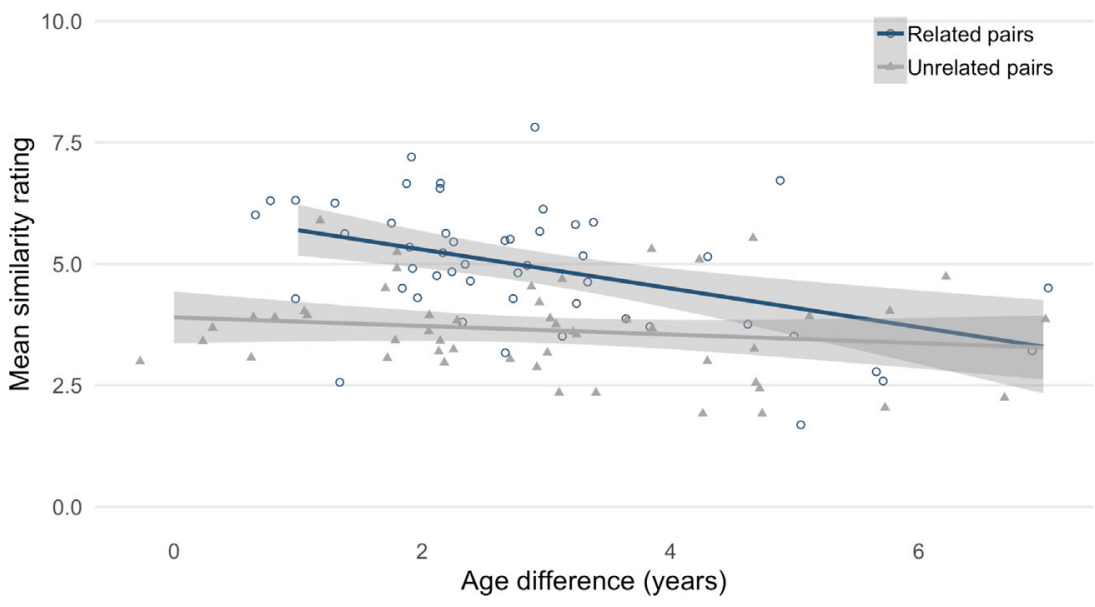

Figure 2. The interaction between relatedness and age difference on similarity judgments.

\section{Similarity task}

We used a linear mixed model to predict similarity judgments from actual relatedness (effect coded as related $=+0.5$ and unrelated $=-0.5$ ), sex difference (effect coded as same-sex $=-0.5$ and opposite-sex $=+0.5$ ), age difference (centered), and all possible interactions in the similarity task.

The analysis revealed a main effect of relatedness, $\beta=1.27, S E=0.21, t=6.06, p<.001$, odds ratio $=3.56$, whereby actually related pairs received higher mean similarity judgments than unrelated pairs. We also found a main effect of age difference within a pair, $\beta=-0.22, S E=0.06, t=-3.4, p<.001$, odds ratio $=0.80$, whereby the greater the age difference within the pair, the lower the mean similarity judgment they received. The main effect of age difference was qualified by an interaction with relatedness, $\beta=-0.27$, $S E=0.13, t=-2.1, p=0.038$, odds ratio $=0.76$, whereby the greater the age difference within sibling pairs, the lower the mean similarity judgment they received. Age difference did not affect mean similarity judgments of unrelated pairs (Figure 2). The main effect of sex difference was not significant, $\beta=-0.38$, $S E=0.21, t=-1.82, p=0.072$, odds ratio $=0.68$, but in the direction of same sex pairs receiving higher mean similarity judgments than opposite sex pairs. There was no significant interaction between sex difference and relatedness, $\beta=-0.79, S E=0.42, t=-1.89, p=0.062$, odds ratio $=0.45$, sex difference, and age difference, $\beta=-0.13, S E=0.13, t=-0.98, p=0.329$, odds ratio $=0.88$, or relatedness, sex difference, and age difference, $\beta=-0.32, S E=0.26, t=-1.24, p=0.217$, odds ratio $=0.73$.

\section{Thresholding}

The outcome variables analyzed were in their original recorded format, with the kinship outcome variable being a binary "related" or "unrelated" response and the similarity outcome variable being on a scale 


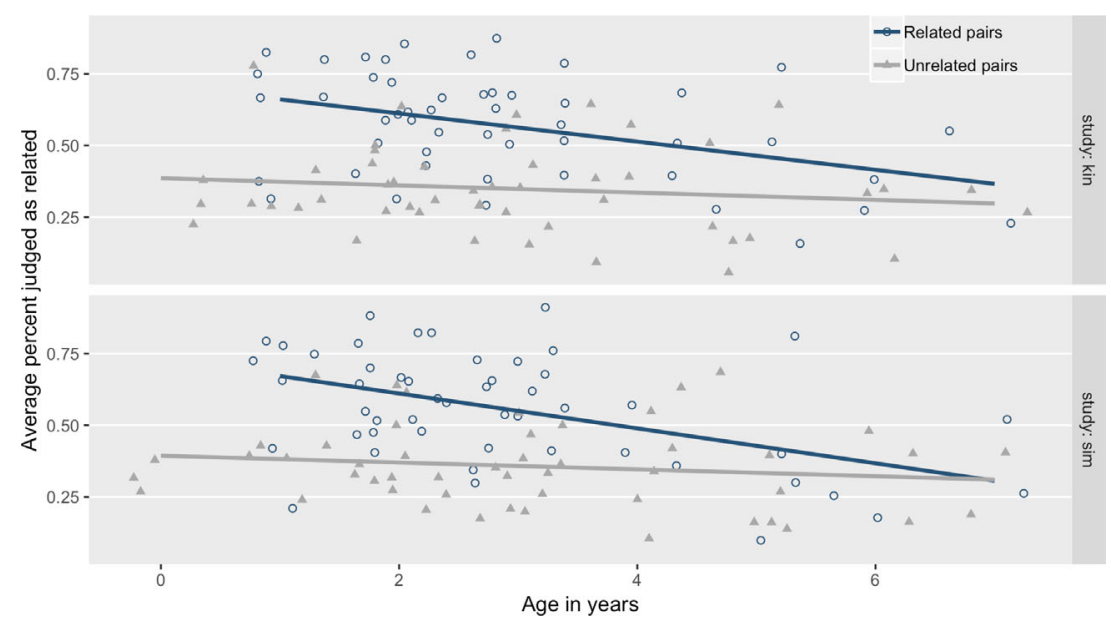

Figure 3. Three-way interaction between relatedness, age, and study task.

from 0 (not very similar) to 10 (very similar). These outcome variables are not on the same scale, therefore they needed to be further processed to be able to directly compare the results from the two tasks. In a thresholding process, the similarity ratings ranging from 0 to 10 were transformed into a binary outcome variable following Maloney and Dal Martello (2006). This goal was achieved by first calculating the mean percentage of each 0 to 10 rating for related and unrelated pairs separately. Then, we calculated the log likelihood ratio of receiving each score depending on the pair being related or unrelated by dividing the related by the unrelated mean percentage calculated before. These data were then entered into a linear model to obtain the beta coefficient (4.2), which indicates the point at which similarity ratings can be divided into binary ratings. This means that ratings over 4.2 were recoded as "related ratings" in the subsequent threshold analysis and ratings under 4.2 were recoded as "unrelated ratings."

\section{Threshold model}

We could then directly test whether effects of sex and age were significantly different between tasks using a logistic mixed model. Moreover, we could determine whether the tasks differed in participants' kin recognition accuracy. This is an additional analysis model that was not included in the original pre-registration.

The analysis revealed a main effect of relatedness, $\beta=1.11, S E=0.17, z=, p<.001$, odds ratio $=3.03$, whereby related pairs were accurately judged as being related, and unrelated pairs were accurately judged as being unrelated. There was no main effect of study task, $\beta=-0.1, S E=0.13, z=-0.75, p=0.451$, odds ratio $=0.90$, and no interaction between task and relatedness, $\beta=-0.01, S E=0.08, z=-0.1$, $p=0.92$, odds ratio $=0.99$, indicating that both similarity ratings and kinship judgments were equally accurate in detecting actual relatedness. There was no significant interaction between task and sex difference, $\beta=-0.01, S E=0.08, z=-0.13, p=0.898$, odds ratio $=0.99$, or task and age difference, $\beta=-0.05$, $S E=0.02, z=-1.91, p=0.056$, odds ratio $=0.95$. This finding indicates that effects of age and sex difference did not differ between the two tasks. There was, however, a significant three-way interaction between relatedness, age difference and study task, $\beta=-0.1$, $S E=0.05, z=-2.04, p=0.041$, odds ratio $=0.90$, whereby age differences influenced all judgments but similarity judgments of unrelated pairs (Figure 3).

Across both tasks, there was an interaction between relatedness and sex differences, $\beta=-0.69, S E=0.33$, $\mathrm{z}=-2.06, p=0.04$, odds ratio $=0.50$, whereby samesiblings were judged to be related more often than opposite sex siblings. Sex differences did not influence kinship judgments of unrelated pairs (Figure 4).

\section{Discussion}

Kinship detection was equally accurate in the kinship and the similarity tasks. Participants labeled siblings correctly as related in the kinship task and gave significantly higher similarity ratings to siblings in the similarity task. However, we did find a three-way interaction of study task, relatedness, and age differences. This finding means that, although we found a general decrease in kinship judgments and similarity ratings with increasing age differences within the pairs, in the similarity task this interaction was qualified by relatedness. Namely, participants gave lower similarity ratings to siblings when they were further apart in age, but this was not true for unrelated face pairs. In the kinship task, age difference had a similar effect on 


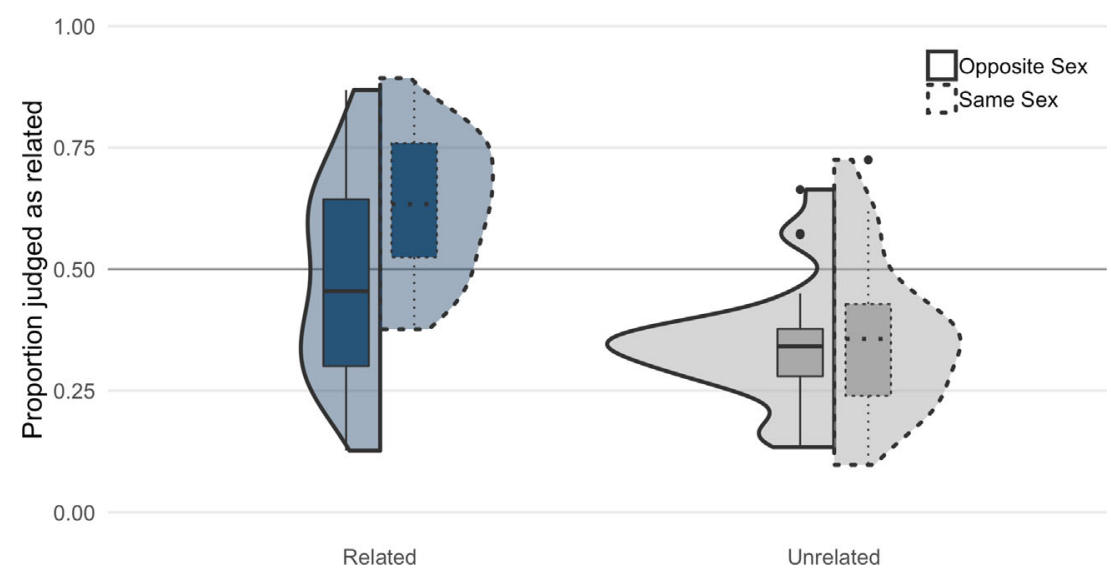

Figure 4. The interaction between relatedness and sex difference on proportion of face pairs judged as related.

kinship judgments of both siblings and unrelated pairs. This finding suggests that similarity ratings and kinship judgments are not necessarily synonymous; they seem to be informed by different information, especially with regard to the age of the stimulus faces. Sex differences affected judgments equally across tasks in the threshold model, whereby same sex siblings were judged to be related more often than opposite sex siblings and received higher similarity scores than opposite sex siblings. Unrelated pairs' kinship judgments and similarity ratings were not affected by sex differences within the pair.

Kinship is a well-defined biological concept, whereas similarity is a relatively vague concept with criteria that vary depending on the context and information available to make similarity judgments. The similarity between objects is a function of the combination of the measures of their common and distinctive features (Tversky, 1977). In the current study, common and distinctive features may include age, sex, and kinship, although their influence may vary depending on context and the combination of information provided. This may explain some of the findings here, for example, the finding that participants gave lower similarity ratings to siblings when they were further apart in age, which was not the case for unrelated pairs.

The two previous studies found that the similarity and kinship task are correlated, $\mathrm{r}=.92$ in Maloney and Dal Martello, 2006; $r=0.90-0.92$ for twin siblings and $\mathrm{r}=0.50-0.72$ for non-twin siblings in DeBruine et al., 2009. In the current study, we found a comparable correlation coefficient of $r=0.93$, meaning that pairs rated as looking more similar in the similarity task were also judged to be related more often in the kinship task, regardless of actual relatedness. Moreover, Maloney and Dal Martello (2006) found that the mean similarity ratings were a strong linear predictor of the log-odds that a pair was actually related or not. Although DeBruine et al. (2009) found comparable results for masked and unmasked images of twins, and unmasked images of non-twin siblings, they found similarity ratings performed significantly worse in predicting actual relatedness for masked images of non-twin siblings. Our score was comparable with that of Maloney and Dal Martello's (2006). This finding could indicate that, in child siblings, perceived similarity is more closely linked to perceived kinship than in adults, as argued by DeBruine et al. (2009).

However, this finding does not necessarily mean that similarity ratings are synonymous with kinship judgments. Although Maloney and Dal Martello (2006) found no difference in accuracy between tasks in a signal detection analysis, DeBruine et al. (2009) did find a significant difference in task accuracy in the unmasked sibling set. Namely, kinship judgments were more accurate at detecting actual siblings and unrelated pairs than similarity ratings. In the current study, a logistic mixed model showed no significant difference in accuracy between similarity and kinship task. That said, in the similarity task, raters gave significantly lower similarity ratings to siblings with a greater age gap, whereas unrelated pairs' similarity ratings were unaffected. In the kinship task, a greater gap in age decreased perceptions of kinship for both related and unrelated face pairs. Age difference within pairs thus had a different effect on similarity and kinship judgments. This finding supports the notion that similarity ratings and kinship judgments are not influenced by age information in the same way. DeBruine et al. (2009) were not able to draw specific conclusions about the effect of age because their sex and age differences were confound. This is not the case in the current study, where the age difference between same sex pairs (mean age difference in years $=2.77 \pm$ 1.56) and opposite sex pairs (mean age difference in years $=3.2 \pm 1.72$ ) is not significantly different, $p=$ 0.193 .

In conclusion, using a larger stimuli sample of children, we replicated Maloney and Dal Martello's (2006) finding that kinship and similarity ratings are 
highly correlated. We found that the effect of sex was the same on similarity and on kinship judgments, contrary to DeBruine et al (2009). However, we found that the effect of age was different on similarity judgments than on kinship judgments, contrary to Maloney and Dal Martello (2006). Hence, in line with previous work, we conclude that similarity ratings and kinship judgments are closely linked and that similarity ratings to a certain extent track the same cues that inform kinship judgments, but that similarity and kinship judgments are not strictly synonymous. This finding could potentially suggest that perceived similarity and kinship judgments use overlapping cues, but weigh and combine them in different ways, especially age information.

Keywords: allocentric kin recognition, face perception, similarity, resemblance

\section{Acknowledgments}

Supported by the European Research Council (ERC) grant [\#647910] awarded to LMD.

Commercial relationships: none.

Corresponding author: Vanessa Fasolt.

Email: v.fasolt.1@research.gla.ac.uk.

Address: University of Glasgow, 62 Hillhead Street, Glasgow G12 8QBx, UK.

\section{References}

Bates, D., Mächler, M., Bolker, B., \& Walker, S. (2015). Fitting linear mixed-effects models using lme4. Journal of Statistical Software, 67(1), 1-48, https://doi.org/10.18637/jss.v067.i01.

Bressan, P., Bertamini, M., Nalli, A., \& Zanutto, A. (2009). Men do not have a stronger preference than women for self-resemblant child faces. Archives of Sexual Behavior, 38(5), 657-664, https://doi.org/10.1007/s10508-008-9350-0.

DeBruine, L. M. (2002). Facial resemblance enhances trust. Proceedings of the Royal Society of London B: Biological Sciences, 269(1498), 1307-1312, https://doi.org/10.1098/rspb.2002.2034.

DeBruine, L. M. (2004a). Facial resemblance increases the attractiveness of same-sex faces more than other-sex faces. Proceedings of the
Royal Society of London B: Biological Sciences, 271(1552), 2085-2090, https: //doi.org/10.1098/rspb.2004.2824.

DeBruine, L. M. (2004b). Resemblance to self increases the appeal of child faces to both men and women. Evolution and Human Behavior, 25(3), 142-154, https: //doi.org/10.1016/j.evolhumbehav.2004.03.003.

DeBruine, L. M. (2005). Trustworthy but not lust-worthy: Context-specific effects of facial resemblance. Proceedings of the Royal Society of London B: Biological Sciences, 272(1566), 919-922, https://doi.org/10.1098/rspb.2004.3003.

DeBruine, L. M., Jones, B. C., Little, A. C., \& Perrett, D. I. (2008). Social perception of facial resemblance in humans. Archives of Sexual Behavior, 37(1), 64-77, https://doi.org/10.1007/s10508-007-9266-0.

DeBruine, L. M., Smith, F. G., Jones, B. C., Roberts, S. C., Petrie, M., \& Spector, T. D. (2009). Kin recognition signals in adult faces. Vision Research, 49(1), 38-43, https: //doi.org/10.1016/j.visres.2008.09.025.

Krupp, D. B., DeBruine, L. M., \& Barclay, P. (2008). A cue of kinship promotes cooperation for the public good. Evolution and Human Behavior, 29(1), 49-55, https://doi.org/10.1016/j.evolhumbehav.2007.08. 002.

Kuznetsova, A., Brockhoff, P. B., \& Christensen, R. H. B. (2016). LmerTest: Tests in linear mixed effects models. Retrieved from https: //CRAN.R-project.org/package=lmerTest.

Maloney, L. T., \& Dal Martello, M. F. (2006). Kin recognition and the perceived facial similarity of children. Journal of Vision, 6, 1047-1056, https://doi.org/10.1167/6.10.4.

Platek,S. M., Raines, D. M., Gallup, G. G., Jr, Mohamed, F. B., Thomson, J. W., Myers, T.E ., Panyavin, I. S., Levin, S. L., Davis, J. A., Fonteyn, L. C., ... Arigo, D. R. (2004). Reactions to children's faces: Males are more affected by resemblance than females are, and so are their brains. Evolution and Human Behavior, 25(6), 394-405, https: //doi.org/10.1016/j.evolhumbehav.2004.08.007.

$\mathrm{R}$ Core Team. (2017). $R$ : A language and environment for statistical computing. Retrieved from https://www.R-project.org/.

Tversky, A. (1977). Features of similarity. Psychological Review, 84(4), 327. 